\title{
The options available in cognitive behavioral therapy to prevent chronification of insomnia
}

\author{
Tatjana Crönlein • Jürgen Zulley
}

Received: 5 May 2011 / Accepted: 14 June 2011 /Published online: 5 July 2011

(C) European Association for Predictive, Preventive and Personalised Medicine 2011

\begin{abstract}
Insomnia is a very frequent problem among the general population, and it has a high socio-economic impact on health care management. It produces high costs because of absenteeism and accidents, and it is considered to be a risk factor for the development of psychiatric diseases and other medical disorders. However, only a low proportion of insomniacs ever seek professional help. The reasons are still unknown, but most insomniacs probably consider their problem untreatable unless they undergo a therapy with hypnotics. Knowledge about alternative therapy methods beyond sleep medication is scarce, and specialized psychotherapy is not always available. Since dysfunctional behavior and beliefs are considered to be the central perpetuating factors for primary insomnia, the role of educational programs in terms of prevention and treating mild forms of insomnia is discussed. These workshops could be a part of a step-by-step treatment of insomnia as has been proposed recently by Espie.
\end{abstract}

Keywords Insomnia $\cdot$ Cognitive behavioral therapy $\cdot$ Health management $\cdot$ Sleep $\cdot$ Prevention $\cdot$ Risk factors

T. Crönlein $\cdot$ J. Zulley

Department of Psychiatry,

University of Regensburg,

Universitaetsstraße 84,

93042 Regensburg, Germany

T. Crönlein $(\triangle)$

Department of Psychiatry,

University of Regensburg,

Bezirksklinikum, Universitätsstraße 84,

93042 Regensburg, Germany

e-mail: tatjana.croenlein@medbo.de

\section{Insomnia, prevalence and risk factors}

Disturbed sleep is a very frequent problem among the general population. Prevalence varies depending on which criteria and assessment methods are used [1]. However, symptoms of disturbed sleep are reported by at least one fifth of the general population [2]. The prevalence of insomnia symptoms varies between $19 \%$ and $29 \%$ among Europeans [3]. Narrowing down the criteria to the point at which insomnia becomes a clinical problem reduces the prevalence to $10 \%$ [4-6]. The general criteria for insomnia according to the International Classification of Sleep Disorders are mainly: Difficulty initiating or maintaining sleep or waking up too early and signs of daytime impairment related to nonrestorative sleep, such as fatigue, energy reduction or worries about sleep [7].

There are some risk factors for developing insomnia. A recent survey showed that nocturnal awakenings increased with age: $52 \%$ of the persons older than 65 reported nocturnal awakenings in contrast to $16 \%$ of adolescents [8]. A further risk factor for having insomnia is being female and single $[8,9]$ and living in large cities [9]. Also, there seems to be a correlation between low social economic status and insomnia [10], as well as shift work [11].

\section{Socio-economic relevance of insomnia for the societies}

The problem of insomnia beyond its subjective harm is its socio-economic relevance for society due to the high costs of sick leave or accidents [12, 13]. Annual costs for 
insomnia have been estimated to be more than 90 billion dollars [14]. A further problem is the impact insomnia has on the development of further psychiatric disorders such as depression [15]. It has been shown that sleep-disturbed people have a higher risk of developing depression [4]. Recent studies have shown that intervention for insomnia either pharmacologic [16] or with Cognitive Behavior Therapy for Insomnia (CBT-I) [17] may reduce the symptoms of depression. While it is postulated that a depressive mood is part of the psychopathology of insomnia [18], some new data suggest a direct effect of sleep loss by emotional regulation [19]. The role of insomnia in developing depression is not clearly understood until now, and the role of disturbed sleep in the context of psychiatric illness remains a field of research [20,21]. However, the treatment of insomnia especially regarding its role as a link to developing other diseases has an increasing value in health care management.

\section{Self-treatment methods or medication?}

How do sleep-disturbed people treat insomnia? It is interesting that although there is a high rate of insomnia among the population, only a few are seeking professional help [22]. Most people seem to treat their disturbed sleep by watching TV, drinking alcohol or using non-prescription medication [23]. The proportion of insomniacs using prescriptions seems to be rather low and ranges from $2.6 \%$ [24] to $11 \%$ in France [25]. The reason for this low usage of professional medical help may be a negative attitude towards pharmacologic therapy among insomnia patients and therefore a low acceptance [26]. The negative effects such as addiction, hangover, dose escalation and side effects for other diseases still complicate the prescription of hypnotics, especially in older persons [27-29]. Another reason is the short duration (four weeks) of the recommended intake of hypnotics. Despite newer data showing that long-term use does not lead to dose escalation [30], a study showing an average duration of use for prescribed medication in insomnia patients of 4 years did not yield optimal drug efficacy in most patients [31].

\section{Current approaches in treating insomnia}

In summary we have a high prevalence of insomnia among the general population, which produces high costs for society. On the other hand, we have a rather low proportion of people with insomnia that seek medical help. Since age is a serious risk factor for developing insomnia and since there will be a higher proportion of elderly in our industrialized society in the future, the effective treatment of insomnia is a current challenge for health care management. What possible options are available to reduce the prevalence of insomnia among the general population?

\section{Perpetuating factors of insomnia as target symptoms for behavior therapy}

In order to evaluate possible preventive and therapeutic options for insomnia, it is worth looking at its perpetuating factors. It is evident by now that primary insomnia may exist without any underlying psychiatric or other organic sleep disorders. In order to explain the perpetuation of disturbed sleep, experts have found that psychological factors are responsible. These models are based on observations of daytime performance in people with insomnia. Symptoms of sleep deprivation are not entirely present in insomniacs [32]. Measuring daytime performance with psychomotor tests, insomniacs did not display results that correspond to their subjective perception of daytime fatigue [33]. This lack of objective fatigue in insomniacs was explained by hyperarousal $[34,35]$. Several studies have shown that this heightened arousal is evident in physiological and psychological parameters [36]. Hyperarousal is also evident in presleep EEG, which is responsible for sleep misperception [37]. Enhanced cognitive rumination, which is a typical symptom in insomniacs, could be explained by hyperarousal. In the course of psychological research, several models of persistent primary insomnia have been described [18, 37, 38]. The main assumption is that insomnia may exist as an interplay of psychological factors such as irrational and dysfunctional beliefs [39], erratic sleep-wake behavior, an enhanced mode of self-observation [38] and a hyperarousal that prevents sufficient relaxation for the process of falling asleep [37].

The role of irrational beliefs in this context as a perpetuating factor has been examined in several studies $[39,40]$. In comparison to healthy controls, insomniacs are much more worried about the consequences of bad sleep, especially since they believe that insomnia may be the cause for bodily and physical damage [39]. Also, insomniacs often believe in dysfunctional sleep-promoting behavior in the hope that it will ensure sleep [39]. Prolonged bedtimes are the most prominent errors made by insomniacs. Since insomnia patients experience a long wake time in bed, they try to compensate sleep loss with a longer period of rest. In consequence, bedtime hours longer than 8 or more hours lead to an impairment of sleep continuity and to prolonged wake time at night. Patients notice this loss of sleep continuity and normally react with frustration. Since they usually tend to avoid everything that might worsen sleep (in this case stress because of short bedtimes), they stick to their 
habits. Dysfunctional remarks such as "I am a bad sleeper" reinforce their insomnia.

\section{Cognitive behavior therapy for insomnia}

For treating insomnia a variety of therapeutic strategies already exist. As far as hypnotics is concerned, especially Benzodiazepine receptor agonists seem to be very effective in treating insomnia [41, 42]. In addition to the pharmacological treatment that has been elaborated in the past 20 years, a specialized behavior therapy form has been established, which is called cognitive behavior therapy for insomnia (CBT-I). CBT-I has been developed on the basis of the understanding that primary insomnia is a conditioned disorder [43, 44]. This form of insomnia is called psychophysiological insomnia. If disturbed sleep in insomnia is a result of dysfunctional thinking and behavior, an effective way to improve sleep would be to change these thoughts and behaviors. It has been shown that CBT-I reduces irrational thoughts and that these improvements correlated with subjective and objective improvement of sleep [40]. Identifying and changing dysfunctional thoughts, therefore, has an important impact on the course of insomnia and it is part of the central educative elements of CBT-I [44]. One important feature of this module is that it can be performed using different media (e.g. books, the Internet) and it is not necessary to have direct contact to a psychotherapist. However, monitoring the effects of altering dysfunctional beliefs still needs therapeutic aid.

Table 1 shows the most important modules of CBT-I.

The main module is sleep restriction, which was first introduced by Spielman et al. [45] and is based on the hypothesis that prolonged bedtime hours are one of the factors that perpetuate insomnia. Patients are instructed to keep certain bedtimes that are restricted in duration to $6 \mathrm{~h}$. Sleep is not allowed anywhere else or at any other time. By restricting allowed sleep to a short period at night, sleep pressure is built up, resulting in improved sleep quality.

Table 1 Methods of cognitive behavior therapy for insomnia

\begin{tabular}{lc}
\hline Method & Treatment goal \\
\hline Sleep restriction & $\begin{array}{c}\text { Improving sleep continuity by } \\
\text { shortening bedtime hours } \\
\text { Redefining the emotional value } \\
\text { of the sleep environment by } \\
\text { only allowing sleep in bed } \\
\text { Altimulus control } \\
\text { Sleep education } \\
\text { attitudes of dysfunctional } \\
\text { beliefs regarding sleep } \\
\text { in order to reduce } \\
\text { sleep-related fears } \\
\text { Reduction of hyperarousal }\end{array}$ \\
\hline
\end{tabular}

Bootzin first saw a relationship between insomnia and the sleep environment as a discriminative stimulus for sleep [46]. Stimulus control therapy is a very effective strategy in reducing anticipatoric anxiety and improving sleep [47]. It is recommended by AASM as the standard non-pharmacological treatment for insomnia [48]. The technique is based on the idea of conditioned sleeplessness. If insomniacs experience disturbed sleep in bed again and again, the bedroom environment may develop into a stimulus that is incompatible with sleep, hence with an arousal reaction. Insomnia patients typically report that they may be very tired, but once they have gone to bed, they become more and more awake. This is the result of conditioned awakening in surroundings that should actually be a discriminative stimulus for sleep.

A third technique is relaxation therapy, since cognitive and physiological hyperarousal is a central aspect in insomnia. A variety of relaxation techniques are used, for example progressive muscle relaxation (PMR), autogenic training, meditation or biofeedback. It is important that the patient feels comfortable with the special relaxation technique.

\section{Effectiveness of CBT}

Non-pharmacological strategies in the treatment of insomnia have their own history. Since Bootzin and Spielman tried to improve sleep quality with behavior therapy modules, a compelling number of studies have been carried out to prove the effectiveness of CBT-I. Studies so far show that CBT-I is more effective than no treatment $[49,50]$ and than PMR and Placebo [51]. The advantage of CBT is its long-term effect beyond the end of therapy [52-54]. This is a very promising feature of CBT, since the long-term use of hypnotics remains a matter of debate. The fact that CBT-I has an enduring positive effect strengthens the hypothesis that sleep improvement is a result of a learning process. Once patients have an insight into dysfunctional behavior and once they are able to alter behavior patterns that are incompatible with sleep, sleep may improve. Patients are able to regain control over the process of sleep.

Meanwhile several meta-analyses have shown its effectiveness, even if insomnia is associated with other psychiatric disorders or medical illnesses [54-56]. However, the disadvantage is the need for an elaborated therapist. The current issue in insomnia therapy management, therefore, is not whether but how to transport therapy tools to the people who need them.

\section{Learning how to sleep}

At this point the learning mechanism of CBT-I turns out to be valuable. If the alternation between dysfunctional and 
sleep-incompatible behavior is the basic mechanism, it seems reasonable to offer educational programs. Educational programs have several advantages. First, they can be applied with different media (e.g. Internet, books). Second, they can be developed according to the current standards of therapy, and third, they reach a wide range of people. Educational programs could be offered in companies or factories, for example, for shift workers. Programs could be adapted for schoolchildren or the needs of older people.

There are some data that already exist about self-help programs and short interventions, such as workshops. Zulley et al. offered an educational program supported by a German health insurance company. The focus of the seminar lies in lectures on normal sleep and sleep disorders. Therapy methods, such as bedtime restriction and relaxation techniques, are taught. Changing dysfunctional beliefs was also a central aspect of these educational programs. The class was open to anyone who was interested. No initial examination was done. However, as was expected, mostly sleep-disturbed people participated. A follow-up evaluation was done with 167 participants after 3 months. The sample consisted of 110 women and 57 men between the ages of 21-88 years, with a mean of 54 years. Two days of education had a positive effect on the perpetuating factors of insomnia: The majority changed their sleep habits. Of the $63.5 \%$ of participants with a previous consumption of hypnotics, $80 \%$ reported a reduction in consumption, with $20 \%$ of them having stopped taking sleep medications. An improvement of sleep 3 months after the seminar was found in $79.6 \%$. This outcome shows the beneficial effect of such a seminar, which may contribute to the prevention of sleep disorders. The outcome is similar to a recently published trial in London, where sleep-disturbed volunteers were educated in workshops. A 1-day workshop was proven to be effective in the reduction of insomnia symptoms and depression [57].

\section{Options of applying CBT-I}

Espie [58] proposed a step-by-step therapy for insomniacs. Self-administered CBT-I on the entry level could be helpful in insomnia, as has been shown before [59]. Although the effects at this therapy level are modest, [60] they might be useful in an integrated step-by-step therapy algorithm. A Canadian group showed that improvement could be achieved in people with insomnia simply by distributing educational material (6 booklets) and with telephone consulting [61]. As has been suggested by Espie, a small number of CBT-I interventions could be done by nurses. For more difficult and complex cases of insomnia patients, CBT-I could be done by psychotherapists in a setting with standardized number of therapy hours. More research is necessary to evaluate the procedures for referrals in the health care system.

\section{Conclusions and outlook}

Insomnia is not always insomnia. It might exist as a symptom as well as a special sleep disorder or a complicated psychiatric disorder with comorbid conditions. In any case, insomnia is a socio-economic risk factor. Elaborated CBT-I therapy programs may be too difficult to apply broadly, since they need special initial diagnostic procedures and qualified psychotherapists. A step-by-step treatment of insomnia may reduce its prevalence, especially in mild forms of insomnia.

Educative modules of CBT-I (for example information about normal sleep and the perpetuating factors of insomnia including maladaptive sleep behavior) could be offered broadly in several ways (insomnia lectures in schools or senior citizens homes, DVD, etc.). In this way the development of dysfunctional beliefs about sleep ("I must have at least 7 h of sleep") and behavior could be prevented. Primary insomnia as a sleep disorder should be diagnosed and treated in ambulatory settings, general practioneers or by specialized therapists. On this health care level a differentiation between insomnia and other comorbid psychiatric disorders is important. CBT-I can be offered for patients in an ambulatory setting in six to eight weekly sessions or in a weekend workshop. These sessions should be offered by psychotherapists. Patients who are difficult to treat, who have problems to dismiss their hypnotic medication or show a severe psychiatric comorbid disorders could be treated in a standardized program for CBT-I either in a psychiatric clinic [62] or a similar setting.

Further research is necessary to show the effectiveness in gradually tailoring of CBT-I for different forms of insomnia.

\section{References}

1. Lichstein KL, Durrence HH, Taylor DJ, Bush AJ, Riedel BW. Quantitative criteria for insomnia. Behav Res Ther. 2003;41 (4):427-45.

2. Ohayon MM, Roth T. Place of chronic insomnia in the course of depressive and anxiety disorders. J Psychiatr Res. 2003;37(1):9-15.

3. Leger D, Guilleminault C, Dreyfus JP, Delahaye C, Paillard M. Prevalence of insomnia in a survey of 12,778 adults in France. J Sleep Res. 2000;9(1):35-42.

4. Ford DE, Kamerow DB. Epidemiologic study of sleep disturbances and psychiatric disorders. An opportunity for prevention? JAMA. 1989;262(11):1479-84.

5. Ancoli-Israel S, Roth T. Characteristics of insomnia in the United States: results of the 1991 National Sleep Foundation Survey. I. Sleep. 1999;22 Suppl 2:S347-53.

6. Ohayon MM, Roth T. What are the contributing factors for insomnia in the general population? J Psychosom Res. 2001;51 (6):745-55.

7. American Academy of Sleep Medicine: The Internation Classification of Sleep Disorders. Westchester, Illinois, 2005.

8. Ohayon MM. Nocturnal awakenings and difficulty resuming sleep: their burden in the European general population. J Psychosom Res. 2010;69(6):565-71. 
9. Hajak G. Epidemiology of severe insomnia and its consequences in Germany. Eur Arch Psychiatry Clin Neurosci. 2001;251(2):49-56.

10. Hatoum HT, Kania CM, Kong SX, Wong JM, Mendelson WB. Prevalence of insomnia: a survey of the enrollees at five managed care organizations. Am J Manag Care. 1998;4(1):79-86.

11. Ohayon MM, Smolensky MH, Roth T. Consequences of shiftworking on sleep duration, sleepiness, and sleep attacks. Chronobiol Int. 2010;27(3):575-89.

12. Leger D, Bayon V. Societal costs of insomnia. Sleep Med Rev. 2010;14(6):379-89.

13. Novak M, Mucsi I, Shapiro CM, Rethelyi J, Kopp MS. Increased utilization of health services by insomniacs - an epidemiological perspective. J Psychosom Res. 2004;56(5):527-36.

14. Stoller MK. Economic effects of insomnia. Clin Ther. 1994;16 (5):873-97.

15. Franzen PL, Buysse DJ. Sleep disturbances and depression: risk relationships for subsequent depression and therapeutic implications. Dialogues Clin Neurosci. 2008;10(4):473-81.

16. Krystal A, Fava M, Rubens R, Wessel T, Caron J, Wilson P, et al. Evaluation of eszopiclone discontinuation after cotherapy with fluoxetine for insomnia with coexisting depression. J Clin Sleep Med. 2007;3(1):48-55.

17. Manber R, Edinger JD, Gress JL, San Pedro-Salcedo MG, Kuo TF, Kalista T. Cognitive behavioral therapy for insomnia enhances depression outcome in patients with comorbid major depressive disorder and insomnia. Sleep. 2008;31(4):489-95.

18. Harvey AG. A cognitive model of insomnia. Behav Res Ther. 2002;40(8):869-93.

19. Yoo SS, Gujar N, Hu P, Jolesz FA, Walker MP. The human emotional brain without sleep - a prefrontal amygdala disconnect. Curr Biol. 2007;17(20):R877-8.

20. Pigeon WR, Hegel M, Unutzer J, Fan MY, Sateia MJ, Lyness JM, et al. Is insomnia a perpetuating factor for late-life depression in the IMPACT cohort? Sleep. 2008;31(4):481-8.

21. Matteson-Rusby SE, Pigeon WR, Gehrman P, Perlis ML. Why treat insomnia? Prim Care Companion J Clin Psychiatry 2010;12 (1): $\mathrm{CC}$.

22. Pires ML, Edito-Silva AA, Mello MT, Pompeia SG, Tufik S. Sleep habits and complaints of adults in the city of Sao Paulo, Brazil, in 1987 and 1995. Braz J Med Biol Res. 2007;40(11):1505-15.

23. Ohayon MM. Epidemiology of insomnia: what we know and what we still need to learn. Sleep Med Rev. 2002;6(2):97-111.

24. Mellinger GD, Balter MB, Uhlenhuth EH. Insomnia and its treatment. Prevalence and correlates. Arch Gen Psychiatry. 1985;42(3):225-32.

25. Leger D, Guilleminault C, Bader G, Levy E, Paillard M. Medical and socio-professional impact of insomnia. Sleep. 2002;25 (6):625-9.

26. Morin CM, Gaulier B, Barry T, Kowatch RA. Patients' acceptance of psychological and pharmacological therapies for insomnia. Sleep. 1992;15(4):302-5.

27. Victorri-Vigneau C, Dailly E, Veyrac G, Jolliet P. Evidence of zolpidem abuse and dependence: results of the French Centre for Evaluation and Information on Pharmacodependence (CEIP) network survey. Br J Clin Pharmacol. 2007;64(2):198-209.

28. Bachman DL. Sleep disorders with aging: evaluation and treatment. Geriatrics. 1992;47(9):53-61.

29. Kamel NS, Gammack JK. Insomnia in the elderly: cause, approach, and treatment. Am J Med. 2006;119(6):463-9.

30. Roehrs TA, Randall S, Harris E, Maan R, Roth T. Twelve months of nightly zolpidem does not lead to dose escalation: a prospective placebo-controlled study. Sleep. 2011;34(2):207-12.

31. Krakow B, Ulibarri VA, Romero E. Persistent insomnia in chronic hypnotic users presenting to a sleep medical center: a retrospective chart review of 137 consecutive patients. J Nerv Ment Dis. 2010;198(10):734-41.
32. Bonnet MH, Arand DL. The consequences of a week of insomnia. Sleep. 1996;19(6):453-61.

33. Seidel WF, Ball S, Cohen S, Patterson N, Yost D, Dement WC. Daytime alertness in relation to mood, performance, and nocturnal sleep in chronic insomniacs and noncomplaining sleepers. Sleep. 1984;7(3):230-8.

34. Bastien CH, St-Jean G, Morin CM, Turcotte I, Carrier J. Chronic psychophysiological insomnia: hyperarousal and/or inhibition deficits? An ERPs investigation. Sleep. 2008;31(6):887-98.

35. Bonnet MH, Arand DL. Insomnia, metabolic rate and sleep restoration. J Intern Med. 2003;254(1):23-31.

36. Riemann D, Spiegelhalder K, Feige B, Voderholzer U, Berger M, Perlis M, et al. The hyperarousal model of insomnia: a review of the concept and its evidence. Sleep Med Rev. 2010;14(1):19-31.

37. Perlis ML, Giles DE, Mendelson WB, Bootzin RR, Wyatt JK. Psychophysiological insomnia: the behavioural model and a neurocognitive perspective. J Sleep Res. 1997;6(3):179-88.

38. Espie CA, Broomfield NM, MacMahon KM, Macphee LM, Taylor LM. The attention-intention-effort pathway in the development of psychophysiologic insomnia: a theoretical review. Sleep Med Rev. 2006;10(4):215-45.

39. Morin CM, Stone J, Trinkle D, Mercer J, Remsberg S. Dysfunctional beliefs and attitudes about sleep among older adults with and without insomnia complaints. Psychol Aging. 1993;8(3):463-7.

40. Edinger JD, Wohlgemuth WK, Radtke RA, Marsh GR, Quillian RE. Does cognitive-behavioral insomnia therapy alter dysfunctional beliefs about sleep? Sleep. 2001;24(5):591-9.

41. Dundar Y, Boland A, Strobl J, Dodd S, Haycox A, Bagust A, et al. Newer hypnotic drugs for the short-term management of insomnia: a systematic review and economic evaluation. Health Technol Assess. 2004;8(24):iii-125.

42. Sullivan SS. Insomnia pharmacology. Med Clin North Am. 2010;94(3):563-80.

43. Edinger JD, Means MK. Cognitive-behavioral therapy for primary insomnia. Clin Psychol Rev. 2005;25(5):539-58.

44. Belanger L, Savard J, Morin CM. Clinical management of insomnia using cognitive therapy. Behav Sleep Med. 2006;4(3):179-98.

45. Spielman AJ, Saskin P, Thorpy MJ. Treatment of chronic insomnia by restriction of time in bed. Sleep. 1987;10(1):45-56.

46. Bootzin RR. A stimulus control treatment for insomnia. Poc Am Psychol Assoc 1972;395-6.

47. Bootzin RR, Lack L, Wright H. Efficacy of bright light and stimulus control instructions for sleep onset insomnia. Sleep. 1999;22 Suppl 1:53-4.

48. Chesson Jr AL, Anderson WM, Littner M, Davila D, Hartse K, Johnson S, et al. Practice parameters for the nonpharmacologic treatment of chronic insomnia. An American Academy of Sleep Medicine report. Standards of Practice Committee of the American Academy of Sleep Medicine. Sleep. 1999;22(8):1128-33.

49. Morin CM, Kowatch RA, Barry T, Walton E. Cognitive-behavior therapy for late-life insomnia. J Consult Clin Psychol. 1993;61 (1):137-46.

50. Espie CA, Inglis SJ, Tessier S, Harvey L. The clinical effectiveness of cognitive behaviour therapy for chronic insomnia: implementation and evaluation of a sleep clinic in general medical practice. Behav Res Ther. 2001;39(1):45-60.

51. Edinger JD, Wohlgemuth WK, Radtke RA, Marsh GR, Quillian RE. Cognitive behavioral therapy for treatment of chronic primary insomnia: a randomized controlled trial. JAMA. 2001;285(14):1856-64.

52. Morin CM, Colecchi C, Stone J, Sood R, Brink D. Behavioral and pharmacological therapies for late-life insomnia: a randomized controlled trial. JAMA. 1999;281(11):991-9.

53. Backhaus J, Hohagen F, Voderholzer U, Riemann D. Long-term effectiveness of a short-term cognitive-behavioral group treatment for primary insomnia. Eur Arch Psychiatry Clin Neurosci. 2001;251(1):35-41. 
54. Riemann D, Perlis ML. The treatments of chronic insomnia: a review of benzodiazepine receptor agonists and psychological and behavioral therapies. Sleep Med Rev. 2009;13(3):205-14.

55. Morin CM, Culbert JP, Schwartz SM. Nonpharmacological interventions for insomnia: a meta-analysis of treatment efficacy. Am J Psychiatry. 1994;151(8):1172-80.

56. Smith MT, Perlis ML, Park A, Smith MS, Pennington J, Giles DE, et al. Comparative meta-analysis of pharmacotherapy and behavior therapy for persistent insomnia. Am J Psychiatry. 2002;159(1):5-11.

57. Archer M, Brown JS, Idusohan H, Coventry S, Manoharan A, Espie CA. The development and evaluation of a large-scale selfreferral CBT-I intervention for men who have insomnia: an exploratory study. Behav Cogn Psychother. 2009;37(3):239-48.
58. Espie CA. "Stepped care": a health technology solution for delivering cognitive behavioral therapy as a first line insomnia treatment. Sleep. 2009;32(12):1549-58.

59. Van Straten A, Cuijpers P, Smit F, Spermon M, Verbeek I. Self-help treatment for insomnia through television and book: a randomized trial. Patient Educ Couns. 2009;74(1):29-34.

60. Van Straten A, Cuijpers P. Self-help therapy for insomnia: a metaanalysis. Sleep Med Rev. 2009;13(1):61-71.

61. Mimeault V, Morin CM. Self-help treatment for insomnia: bibliotherapy with and without professional guidance. J Consult Clin Psychol. 1999;67(4):511-9.

62. Cronlein T, Hajak G. Nonpharmaceutical methods for treatment of insomnia. Pharm Unserer Zeit. 2007;36(3):222-5. 